\title{
Hole-making technique for the treatment for acute pancreatitis due to placement of a fully covered duodenal metallic stent
}

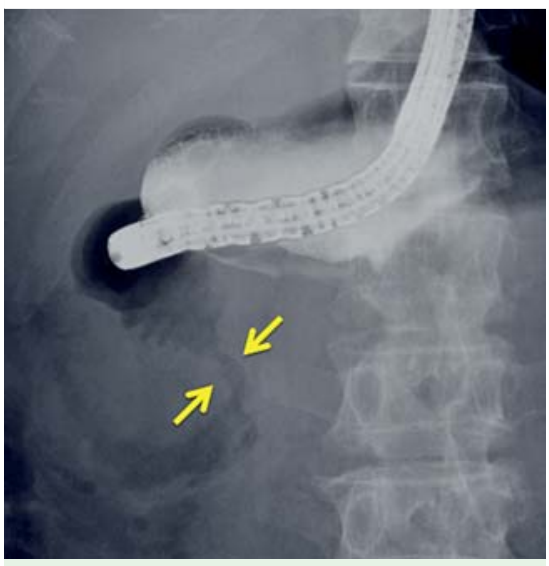

Fig. 1 A duodenal stenosis is apparent (arrows) in a 78-year-old man admitted with vomiting and obstructive jaundice.

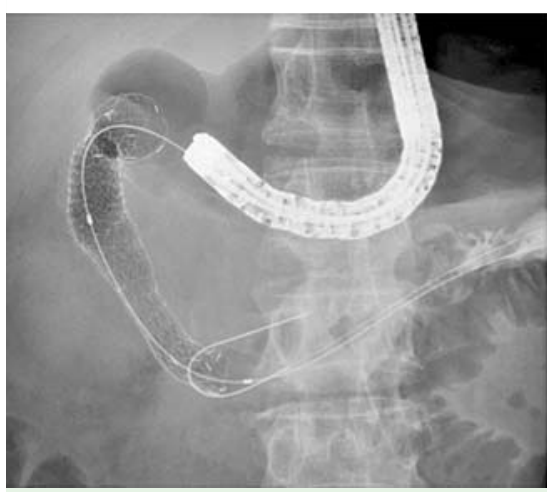

Fig. 2 Duodenal placement of a fully covered metallic stent.

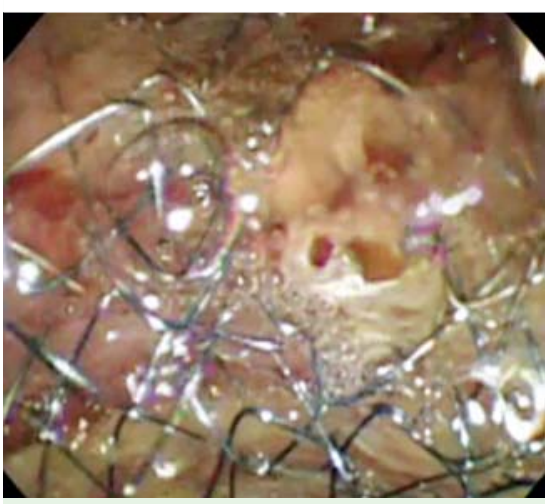

Fig. 5 Pancreatic juice can pass through the hole made with the diathermic dilator.

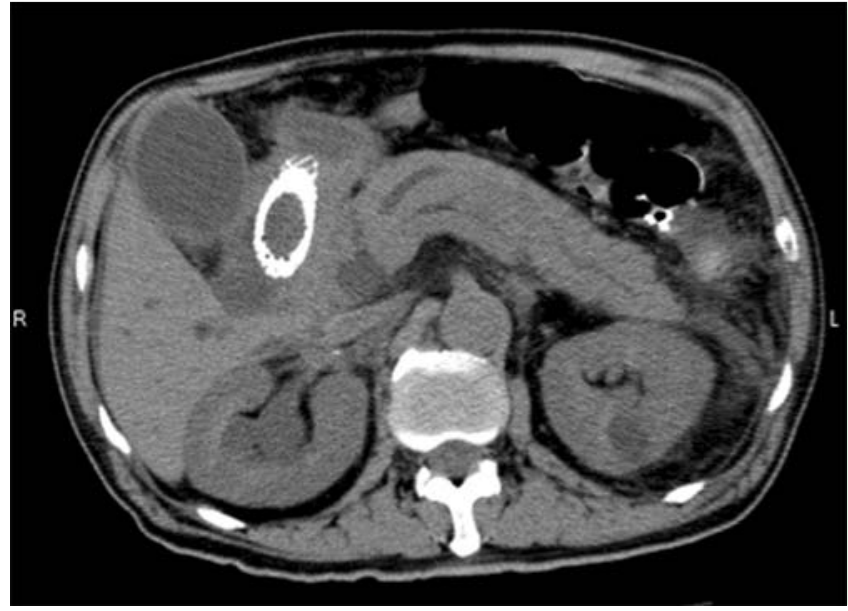

Fig. 3 The main pancreatic duct is dilated.

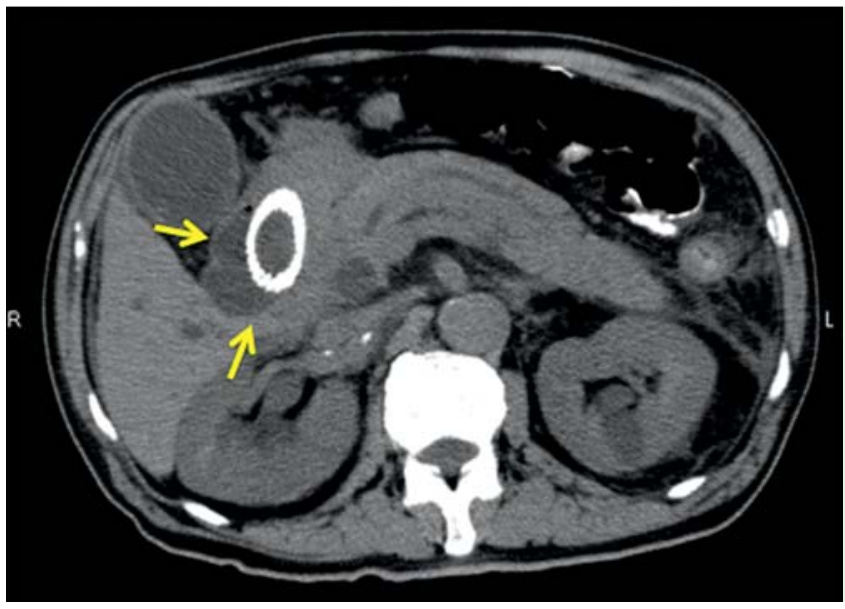

Fig. 4 Fluid is evident around the duodenal metallic stent (arrows).

Duodenal stent placement has been developed as an alternative to surgical gastrojejunostomy [1]. Compared with uncovered metallic stents, duodenal fully covered metallic stents offer advantages, such as the prevention of tumor ingrowth, and thus lower occlusion rates [2]. However, duodenal fully covered metallic stents also have several disadvantages, such as stent migration [3]. Acute pancreatitis is another adverse event associated with duodenal stent placement, occurring at a frequency of approximately $4 \%$ $[4,5]$. In previous reports, acute pancreatitis has been treated conservatively. If a fully covered metallic stent is placed in the duodenum, the frequency of acute pancreatitis may be increased, and the treatment of acute pancreatitis may prove difficult. We report herein a novel treatment for acute pancreatitis due to duode- nal placement of a fully covered metallic stent.

A 78-year-old man was admitted to our hospital because of vomiting and obstructive jaundice. He was undergoing chemotherapy for bladder cancer. Computed tomography showed a duodenal stenosis, attributed to lymph node metastasis. No other site of stenosis was detected, so we attempted duodenal stent placement. First, we advanced the endoscope into the duodenum, and contrast medium was injected. The second part of the duodenum was obstructed ( $\bullet$ Fig. 1 ). Then, we placed a fully covered metallic stent (ComVi, $20 \mathrm{~mm} \times 12 \mathrm{~cm}$; TaeWoong Medical, Seoul, South Korea) in the duodenum (৫ Fig. 2).

Although the vomiting resolved completely, acute pancreatitis developed. Computed tomography showed dilatation of the 


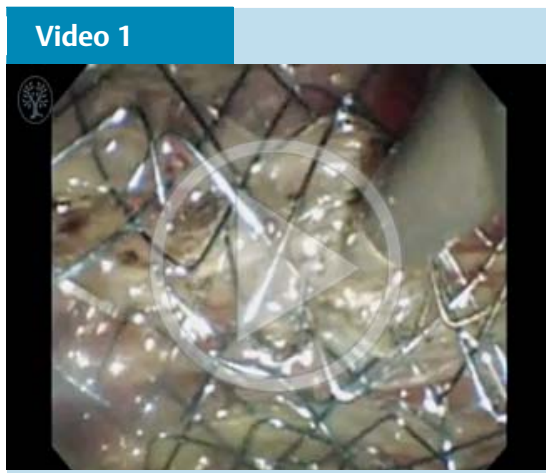

The endoscope is advanced into the duodenal stent. A hole is made with a diathermic dilator, allowing pancreatic juice to pass through.

main pancreatic duct and fluid around the duodenal stent ( $\bullet$ Fig.3, $\bullet$ Fig.4), which were attributed to obstruction of the ampulla of Vater by the duodenal stent. We therefore inserted a diathermic dilator into the duodenal metallic stent and made a hole in the stent, which allowed the flow of pancreatic juice ( $\bullet$ Fig.5, Video 1 ). The acute pancreatitis immediately improved.
Our technique may be useful in cases of acute pancreatitis caused by duodenal placement of a fully covered metallic stent.

Endoscopy_UCTN_Code_TTT_1AO_2AZ

Competing interests: None

Takeshi Ogura, Wataru Takagi, Saori Onda, Tatsushi Sano, Daisuke Masuda, Shinya Fukunishi, Kazuhide Higuchi

2nd Department of Internal Medicine, Osaka Medical College, Osaka, Japan

\section{References}

1 Mauro MA, Koehler RE, Baron TH. Advances in gastrointestinal intervention: the treatment of gastroduodenal and colorectal obstructions with metallic stents. Radiology 2000; 215: 659-669

2 Pan YM, Pan J, Guo LK et al. Covered versus uncovered self-expandable metallic stents for palliation of malignant gastric outlet obstruction: a systematic review and metaanalysis. BMC Gastroenterol 2014; 30: 170

3 Woo SM, Kim DH, Lee WJ et al. Comparison of uncovered and covered stents for the treat- ment of malignant duodenal obstruction caused by pancreaticobiliary cancer. Surg Endosc 2013; 27: 2031-2039

4 Shi-Yi L, Ai-Wu M, Yi-Ping J et al. Placement of duodenal stents across the duodenal papilla may predispose to acute pancreatitis: a retrospective analysis. Diagn Interv Radiol 2012; 18: 360-364

5 Liu SY, Mao AW, Jia YP et al. Placement of a duodenal stents bridge the duodenal papilla may predispose to acute pancreatitis. Hepatogastroenterology 2014; 61: 475-479

\section{Bibliography}

Dol http://dx.doi.org/

10.1055/s-0034-1393146

Endoscopy 2015; 47: E486-E487

(C) Georg Thieme Verlag KG

Stuttgart · New York

ISSN 0013-726X

\section{Corresponding author \\ Takeshi Ogura, MD, PhD}

2nd Department of Internal Medicine

Osaka Medical College

2-7 Daigakuchou

Takatsukishi

Osaka 569-8686

Japan

Fax: +81-527635233

oguratakeshi0411@yahoo.co.jp 